\title{
Parametric and Sliced Causality
}

\author{
Feng Chen and Grigore Roşu \\ Department of Computer Science \\ University of Illinois at Urbana - Champaign, USA \\ ffengchen, grosu\}@uiuc.edu
}

\begin{abstract}
Happen-before causal partial orders have been widely used in concurrent program verification and testing. This paper presents a parametric approach to happen-before causal partial orders. Existing variants of happen-before relations can be obtained as instances of the parametric framework. A novel causal partial order, called sliced causality, is then defined also as an instance of the parametric framework, which loosens the obvious but strict happen-before relation by considering static and dynamic dependence information about the program. Sliced causality has been implemented in a runtime predictive analysis tool for JAVA, named JPREDICTOR, and the evaluation results show that sliced causality can significantly improve the capability of concurrent verification and testing.
\end{abstract}

\section{Introduction}

Concurrent systems are notoriously difficult to verify, test and debug due to their inherent nondeterminism. The happen-before causality, first introduced in [14], provides an effective way to analyze the potential dynamic behaviors of concurrent systems and has been widely used in concurrent program verification and testing [21|16 18 19]9]. Approaches based on happen-before causality extract causal partial orders by analyzing process or thread communication at runtime; the extracted causal partial order can be regarded as an abstract model of the runtime behaviors of the program and thus can be checked against the desired property. This way, one analyzes a class of executions that are characterized by the same causal partial order. Therefore, for verification, the state space to explore can be reduced, while for testing, one can predict potential errors without re-execute the program. Happen-before based approaches are sound (no false alarms) and can handle general purpose properties, e.g., temporal ones.

Several variants of happen-before causalities have been introduced for applications in different domains [14]1619]. Although these notions are similar in principle, there is however no adequate unifying framework for all of them. A proof of soundness has to be re-done for every variant of happen-before causality, which typically involves sophisticate details of the specific domain. This may slow future developments, in particular defining new, or domain-specific causalities. The first contribution of this paper is a parametric framework for causal partial orders, which is purposely designed to facilitate defining and proving correctness of happen-before causalities. The proof of correctness of a happen-before relation is reduced to proving a simple, easy to check closed local property of causal dependence. Existing variants of happen-before relations can be obtained and proved sound as instances of our parametric framework. 
The second contribution of this paper consists of using the above framework to define a novel causal partial order relation, called sliced causality, which aims at improving coverage of analysis without giving up soundness or genericity of properties to check: it works with any monitorable (safety) properties, including regular patterns, temporal assertions, data-races, atomicity, etc. Previous approaches based on happen-before (such as [16 18 19]) extract causal partial orders from analyzing exclusively the dynamic thread communication in executions. Since they consider all interactions among threads, e.g., all reads/writes of shared variables, the obtained causal partial orders are rather restrictive, or rigid, in the sense of allowing a reduced number of linearizations and thus of errors that can be detected. In general, the larger the causality (as a binary relation) the fewer linearizations it has, i.e., the more restrictive it is.

Let us consider a simple and common safety property for a shared resource, that any access should be authenticated. Figure 1 shows a buggy multi-threaded program using the shared resource. The main thread authenticates and then the task thread uses the authenticated resource. They communicate via the flag variable. Synchronization is unnec-

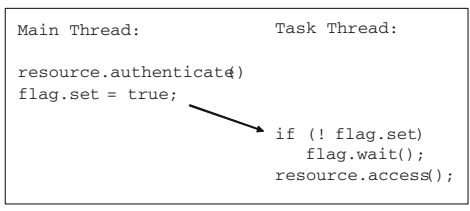

Fig. 1. Multi-threaded execution essary, since only the main thread modifies flag. However, the developer makes a (rather common [7]) mistake by using if instead of while in the task thread. Suppose now that we observed a successful run of the program, as shown by the arrow. The traditional happen-before will not be able to find the bug because of the causality induced by write/read on flag. But since resource.access () is not controlled by if, our technique will be able to correctly predict the violation from the successful execution. When the bug is fixed by replacing if with while, resource.access() will be controlled by while because it is a non-terminating loop; then no violation will be reported by our technique.

In summary, based on an apriori static analysis, sliced causality drastically cuts the usual happen-before causality by removing unnecessary dependencies; this way, a significantly larger number of consistent runs can be inferred and thus analyzed. Experiments show that, on average, the sliced causality relation has about 50\% direct interthread causal dependencies compared to the more conventional happen-before partial order (Section 5). Since the number of linearizations of a partial order tends to be exponential with the size of the complement of the partial order, any linear reduction in size of the sliced causality compared to traditional happen-before relations is expected to increase exponentially the coverage of the corresponding analysis, still avoiding any false alarms. Indeed, the use of sliced causality allowed us to detect concurrency errors that would be very little likely detected using the conventional happen-before causalities.

This paper is organized as follows. Section 2 introduces existing happen-before causalities. Section 3 defines our parametric framework. Section 4 proposes sliced causality. Section 5 discusses the evaluation of sliced causality and Section 6 concludes. Most proofs are omitted because of limited space; they can be found in [4]. 


\section{Happen-Before Causalities}

The first happen-before relation was introduced almost 3 decades ago by Lamport [14], to formally model and reason about concurrent behaviors of distributed systems. Since then, a plethora of variants of happen-before causal partial order relations have been introduced in various frameworks and for various purposes. The basic idea underlying happen-before relations is to observe the events generated by the execution of a distributed system and, based on their order, their type and a straightforward causal flow of information in the system (e.g., the receive event of a message follows its corresponding send event), to define a partial order relation, the happen-before causality. Two events related by the happen-before relation are causally linked in that order.

When using a particular happen-before relation for (concurrent) program analysis, the crucial property of the happen-before relation is that, for an observed execution trace $\tau$, other sound permutations of $\tau$, also called linearizations or linear extensions or consistent runs or even topological sortings in the literature, are also possible computations of the concurrent system. Consequently, if any of these linearizations violates or satisfies a property $\varphi$, then the system can indeed violate or satisfy the property, regardless of whether the particular observed execution that generated the happen-before relation violated or satisfied the property, respectively. For example, [6] defines formulae Definitely $(\varphi)$ and Possibly $(\varphi)$, which hold iff $\varphi$ holds in all and, respectively, in some possible linearizations of the happen-before causality.

The soundness/correctness of a happen-before causality can be stated as follows: given a happen-before causal partial order extracted from a run of the concurrent system under consideration, all its linearizations are feasible, that is, they correspond to other possible execution of the concurrent system. To prove it, one needs to formally define the actual computational model and what a concurrent computation is; these definitions tend to be rather intricate and domain-specific. For that reason, proofs need to be redone in different settings facing different "details", even though they follow conceptually the same idea. In the next section we present a simple and intuitive property on traces, called feasibility, which ensures the desired property of the happen-before causality and which appears to be easy to check in concrete situations.

To show how the various happen-before causalities fall as special cases of our parametric approach, we recall two important happen-before partial orders, one in the context of distributed systems where communication takes place exclusively via message passing, and another in the context of multithreaded systems, where communication takes place via shared memory. In the next section we show that their correctness [2]19] follow as corollaries of our main theorem. In Section 4 we define another happen-before causality, called sliced causality, which non-trivially uses static analysis information about the multithreaded program. The correctness of sliced causality will also follow as a corollary of our main theorem in the next section.

In the original setting of [14], a distributed system is formalized as a collection of processes communicating only by means of asynchronous message passing. A process is a sequence of events. An event can be a send of a message to another process, a receive of a message from another process, or an internal (local) event. 


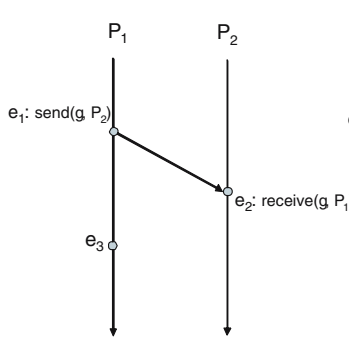

(A) HB in distributed systems

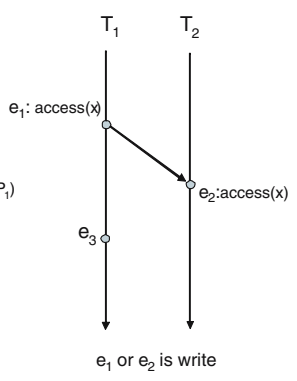

(B) HB in multithreaded systems

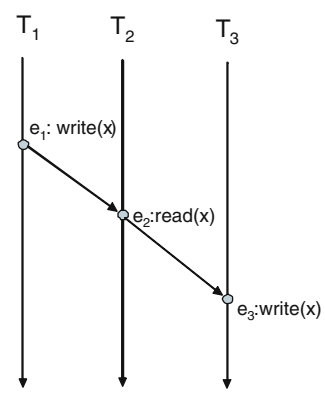

Fig. 2. Happen-before partial-order relations

Fig. 3. Happen-before causality in multi-threaded systems

Definition 1. Let $\tau$ be an execution trace of a distributed system consisting of a sequence of events as above. Let $E$ be the set of all events appearing in $\tau$ and let the happen-before partial order " $\rightarrow$ ” on $E$ be defined as follows:

1. if $e_{1}$ appears before $e_{2}$ in some process, then $e_{1} \rightarrow e_{2}$;

2. if $e_{1}$ is the send and $e_{2}$ is the receive of the same message, then $e_{1} \rightarrow e_{2}$;

3. $e_{1} \rightarrow e_{2}$ and $e_{2} \rightarrow e_{3}$ implies $e_{1} \rightarrow e_{3}$.

A space-time diagram to illustrate the above definition is shown in Figure 2 (A), in which $e_{1}$ is a send message and $e_{2}$ is the corresponding receive message; $e_{1} \rightarrow e_{2}$ and $e_{1} \rightarrow e_{3}$, but $e_{2}$ and $e_{3}$ are not related. It is easy to prove that $(E, \rightarrow)$ is a partial order. The soundness of this happen-before relation, i.e., all the permutations of $\tau$ consistent with $\rightarrow$ are possible computations of the distributed system, was proved in [2] using a specific formalization of the global state of a distributed system. This property will follow as an immediate corollary of our main theorem in the next section.

Happen-before causalities have been devised in the context of multithreaded systems for various purposes. For example, [15,16] propose datarace detection techniques based on intuitive multi-threaded variants of happen-before causality, [19] proposes a happenbefore relation that drops read/read dependencies, and [20] even drops the write/write conflicts but relates each write with all its subsequent reads atomically.

Finding appropriate happen-before causalities for multithreaded systems is a nontrivial task. The obvious approach would be to map the inter-thread communication in a multi-threaded system into send/receive events in some corresponding distributed system. For example, starting a new thread generates two events, namely a send event from the parent thread and a corresponding receive event from the new thread; releasing a lock is a send event and acquiring a lock is a receive event. A write on a shared variable is a send event while a read is a receive event. However, such a simplistic mapping suffers from several problems related to the semantics of shared variable accesses. First, every write on a shared variable can be followed by multiple reads whose order should not matter; in other words, some "send" events now can have multiple corresponding "receive" events. Second, consider the example in Figure 3 Since $e_{3}$ may write a different value into $x$ other than $e_{1}$, the value read at where $e_{2}$ occurs may change if we observe $e_{3}$ after $e_{1}$ but before $e_{2}$ appears. Therefore, $e_{1} e_{3} e_{2}$ may not be a trace of some 
feasible execution since $e_{2}$ will not occur any more. Hence, a causal order between $e_{2}$ and $e_{3}$ should be enforced, which cannot be captured by the original definition in [14].

The various causalities for multithreaded systems address these problems (among others). However, each of them still needs to be proved correct: any sound permutation of events results in a feasible execution of the multithreaded system. If one does not prove such a property for one's desired happen-before causality, then one's analysis techniques can lead to false alarms. We next recall one of the simplest happen-before relations for multi-threaded systems [19]:

Definition 2. Let $\tau$ be an execution of a multithreaded system, let $E$ be the set of all events in $\tau$, and let the happen-before partial order “ $\sim$ " on E be defined as follows:

1. if $e_{1}$ appears before $e_{2}$ in some thread, then $e_{1} \leadsto e_{2}$;

2. if $e_{1}$ and $e_{2}$ are two accesses on the same shared variable such that $e_{1}$ appears before $e_{2}$ in the execution and at least one of them is a write, then $e_{1} \leadsto e_{2}$;

3. $e_{1} \leadsto e_{2}$ and $e_{2} \leadsto e_{3}$ implies that $e_{1} \leadsto e_{3}$.

In Figure2(B), $e_{1} \leadsto e_{2}$ and $e_{1} \leadsto e_{3}$, but $e_{2}$ and $e_{3}$ are not comparable under $\leadsto$.

\section{Parametric Framework for Causality}

We here define a parametric framework that axiomatizes the notion of causality over events and feasibility of traces in a system-independent manner. We show that proving the feasibility of the linearizations of a causal partial order extracted from an execution can be reduced to checking a simpler "closure" local property on feasible traces.

Let Events be the set of all events. A trace $\tau$ is a finite ordered set of (distinct) events $\left\{e_{1}<e_{2}<\cdots<e_{n}\right\}$, usually identified with the Events ${ }^{*}$ word $e_{1} \cdots e_{n}$. Let $\xi_{\tau}=\left\{e_{1}, e_{2}, \ldots, e_{n}\right\}$ be called the alphabet of $\tau$ and $<_{\tau}$ be the total order on $\xi_{\tau}$ induced by $\tau$. Let Traces denote the set of all such traces. Given a set $X$, let $\mathcal{P} O(X)$ denote the set of partial orders defined on subsets of $X$, that is, the set of pairs $(\xi,<)$ where $\xi \subseteq X$ and $<\subseteq \xi \times \xi$ is a partial order.

Definition 3. A causality operator is a partial function $C$ : Traces $\leftrightarrow \mathscr{P} O$ (Events) s.t.:

1. If $C(\tau)=(\xi,<)$, then $\xi=\xi_{\tau}$ and $<\subseteq \subseteq<_{\tau}$; and

2. For any $\tau=\tau_{1} e_{1} e_{2} \tau_{2}$ such that $C(\tau)=(\xi,<)$ with $e_{1} \nless e_{2}, C\left(\tau_{1} e_{2} e_{1} \tau_{2}\right)$ is also defined and equal to $C(\tau)$.

Let $\operatorname{Dom}(C)$ denote the domain of $C$.

Note that condition (ii) in the definition above is closely related to that of trace equivalence introduced by Mazurkiewicz in [11]. However, the theory of Mazurkiewicz traces starts with a given dependency relation on events and considers equivalence of traces according to that fixed dependency, while in our framework, we prefer to associate a separate dependency relation to each trace, assuming that only at runtime we can get enough information about the causality for a given trace; for example, acquiring lock $l$ and writing shared variable $x$ can be or not dependent events, depending on the particular execution of the program. This allows us to be more precise while still using part of the generic Mazurkiewicz trace theory to simplify our correctness proofs.

Any concurrent system can produce only a particular subset of feasible traces, which are in the following relationship with the corresponding causality operator: 
Definition 4. Given a causality operator $C$, a set $\mathcal{F}$ of traces is $C$-feasible iff $C$ is defined on $\mathcal{F}$ and for any $\tau \in \mathcal{F}$ with $C(\tau)=(\xi,<), \mathcal{F}$ contains all the linearizations of $C(\tau)$ (i.e., all traces $\tau^{\prime}$ such that $\xi_{\tau^{\prime}}=\xi$ and $<\subseteq<_{\tau^{\prime}}$ ).

Theorem 1. Dom $(C)$ is $C$-feasible, More precisely, if $C(\tau)=(\xi,<)$ then for any $\tau^{\prime}$, $C\left(\tau^{\prime}\right)=C(\tau)$ if and only if $\xi_{\tau^{\prime}}=\xi$ and $<\underline{\subseteq}<_{\tau^{\prime}}$.

Corollary 1. $\mathcal{F}$ is $C$-feasible iff $C$ is defined on $\mathcal{F}$ and for any $\tau=\tau_{1} e_{1} e_{2} \tau_{2}$ such that $C(\tau)=(\xi,<), e_{1} \nless e_{2}$ implies $\tau_{1} e_{2} e_{1} \tau_{2} \in \mathcal{F}$.

The two variants of happen-before relations discussed in Section 2 can be captured as instances of our parametric framework. For the happen-before relation defined in Definition 1 let Events ${ }_{h b}$ be the set of all the send, receive and internal events.

Corollary 2. For an observed trace $\tau$, any permutation of $\tau$ consistent with $\rightarrow$ is a possible computation of the distributed system.

Proof: Let $C_{h b}$ be the partial function Traces $_{h b} \rightarrow \mathcal{P O}\left(\right.$ Events $\left._{h b}\right)$ with $C_{h b}(\tau)=\left(\xi_{\tau}, \rightarrow\right)$ for any $\tau \in \operatorname{Traces}_{h b}$. Let $\mathcal{F}_{h b}$ be the set of computation traces of the distributed system as defined in [2]. The result follows from Corollary 1, noticing that $C_{h b}$ is a causality operator and $\mathcal{F}_{h b}$ is $C_{h b}$-feasible.

For the happen-before relation in Definition 2, let Events ${ }_{m h b}$ be the set of all the write and read events on shared variables as well as all internal events.

Corollary 3. For an observed trace $\tau$ of a multi-threaded system, any permutation of $\tau$ consistent with $\leadsto$ is a possible execution of the multi-threaded system.

Proof: Let $C_{m h b}$ be the partial function Traces Thb $^{\oplus} \rightarrow \mathcal{P O}\left(\right.$ Events $\left._{m h b}\right)$ with $C_{m h b}(\tau)=$ $\left(E_{\tau}, \leadsto\right)$ for any $\tau \in$ Traces $_{m h b}$. Let $\mathcal{F}_{m h b}$ be the set of traces that are generated by all feasible executions of the multi-threaded system (see, e.g., [19] for a formalization of multi-threaded systems). The result follows from Corollary 1 noticing that $C_{m h b}$ is a causality operator and $\mathcal{F}_{m h b}$ is $C_{m h b}$-feasible.

\section{Sliced Causality}

Without additional information about the structure of the program that generated the event trace $\tau$, the least restrictive causal partial order that an observer can extract from $\tau$ is the one which is total on the events generated by each thread and in which each write event of a shared variable precedes all the corresponding subsequent read events. This is investigated and discussed in detail in [20]. In what follows we show that one can construct a much more general causal partial order, called sliced causality, by making use of dependence information obtained statically and dynamically. Briefly, instead of computing the causal partial order on all the observed events like in the traditional happen-before based approaches, our approach first slices $\tau$ according to the desired property and then computes the causal partial order on the achieved slice; the slice contains all the events relevant to the property, as well as all the events upon which the relevant events depend. This way, irrelevant causality on events is trimmed without 
breaking the soundness of the approach, allowing more permutations of relevant events to be analyzed and resulting in better coverage of the analysis.

We employ dependencies among events to assure the correct slicing. The dependence discussed here somehow relates to program slicing [12], but we focus on finer grained units here, namely events, instead of statements. Our analysis keeps track of actual memory locations in every event, available at runtime, avoiding inter-procedural analysis. Also, we need not maintain the entire dependence relation, since we only need to compute the causal partial order among events that are relevant to the property to check. This leads to an effective vector clock (VC) algorithm ([3]).

Intuitively, event $e^{\prime}$ depends upon event $e$ in $\tau$, written $e \sqsubset e^{\prime}$, iff a change of $e$ may change or eliminate $e^{\prime}$. This tells the observer that $e$ should occur before $e^{\prime}$ in any consistent permutation of $\tau$. There are two kinds of dependence: (1) control dependence, written $e \sqsubset_{c t r l} e^{\prime}$, when a change of the state of $e$ may eliminate $e^{\prime}$; and (2) data-flow dependence, written $e \sqsubset_{\text {data }} e^{\prime}$, when a change of the state of $e$ may lead to a change in the state of $e^{\prime}$. While the control dependence only relates events generated by the same thread, the data-flow dependence may relate events generated by different threads: $e$ may write some shared variable in a thread $t$, which is then read in another thread $t^{\prime}$.

\subsection{Events and Traces}

Events represent atomic steps observed in the execution of the program. In this paper, we focus on multi-threaded programs and consider the following types of events (other types can be easily added): write/read of variables, beginning/ending of function invocations, acquiring/releasing locks, and starts and exits of threads. A statement in the program may produce multiple events. Events need to store enough information about the program state in order for the observer to perform its analysis.

Definition 5. An event is a mapping of attributes into corresponding values. A trace is a sequences of events. We let $\tau, \tau^{\prime}$, etc., denote traces. From now on in the paper, we assume an arbitrary but fixed trace $\tau$ and let $\xi$ denote $\xi_{\tau}$ (recall $\left.\xi_{\tau}=\{e \mid e \in \tau\}\right)$ for simplicity; events in $\xi$ are called concrete events.

For example, one event can be $e_{1}:$ (counter $=8$, thread $=t_{1}$, stmt $=L_{11}$, type $=$ write, target $=a$, state $=1$ ), which is a write on location $a$ with value 1 , produced at statement $L_{11}$ by thread $t_{1}$. One can easily include more information into an event by adding new attribute-value pairs. We use $k e y(e)$ to refer to the value of attribute key of event $e$. The attribute state contains the value associated to the event; specifically, for the write/read on a variable, state $(e)$ is the value written to/read from the variable; for ending of a function call, state $(e)$ is the return value if there is one; for the lock operation, state $(e)$ is the lock object; for other events, state $(e)$ is undefined. To distinguish among different occurrences of events with the same attribute values, we add a designated attribute to every event, counter, collecting the number of previous events with the same attribute-value pairs (other than the counter). This way, all events appearing in a trace can be assumed different. 


\subsection{Control Dependence on Events}

Informally, if a change of state(e) may affect the occurrence of $e^{\prime}$, then we say that $e^{\prime}$ has a control dependence on $e$, and write $e \sqsubset_{c t r l} e^{\prime}$. For example, in Figure 4 the write on $x$ at $S_{1}$ and the write on $y$ at $S_{2}$ have a control dependence on the read on $i$ at $C_{1}$, while the write on $z$ at $S_{3}$ does not have such control dependence. Control dependence occurs inside of a thread, so we first define the total order within one thread:

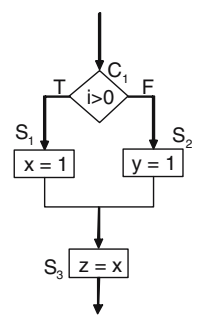

Fig. 4. Control dependence

Definition 6. Let $<$ denote the union of the total orders on events of each thread, i.e., $e<e^{\prime}$ iff thread $(e)=\operatorname{thread}\left(e^{\prime}\right)$ and $e<_{\tau} e^{\prime}$.

The control dependence among events in sliced causality is parametric in a control dependence relation among statements. In particular, one can use off-the-shelf algorithms for classic [8] or for weak [17] control dependence. We chose to use the terminationsensitive control dependence (TSCD) introduced in [5] in our implementation of JPREDICTOR[3]. Nevertheless, all we need to define sliced causality is a function returning the control scope of any statement $C$, say scope $(C)$, which is the set of statements whose reachability depends upon the choice made at $C$, that is, the statements that control depend on $C$, for some appropriate or preferred notion of control dependence. Our approach also regards the lock acquire statement as a control statement that controls all the following statements, since the thread has to wait for the lock to continue its execution.

We assume that any control statement generates either a read event (the lock acquire is regarded as a read on the lock) or no event (the condition is a constant) when checking its condition. For the control statement with a complex condition, e.g., involving function calls and side effects, we can always transform the program to simplify its condition to a simple check of a boolean variable: one can compute the original condition before the control statement, store its result in a fresh boolean variable, and then modify the control statement to check only that variable in its condition.

Definition 7. $e \sqsubset_{c t r l} e^{\prime}$ iff $e<e^{\prime}$, stmt $\left(e^{\prime}\right) \in \operatorname{scope}(\operatorname{stmt}(e))$, and $e$ is "largest" with this property, i.e., there is no $e^{\prime \prime}$ such that $e<e^{\prime \prime}<e^{\prime}$ and $\operatorname{stmt}\left(e^{\prime}\right) \in \operatorname{scope}\left(\operatorname{stmt}\left(e^{\prime \prime}\right)\right)$.

Intuitively, an event $e$ is control dependent on the latest event issued by some statement upon which $\operatorname{stmt}(e)$ depends. For example, in Figure 4 a write of $x$ at $S_{1}$ is control dependent on the most recent read of $i$ at $C_{1}$ and not on previous reads of $i$ at $C_{1}$.

The soundness of analysis based on sliced causality is contingent to the correctness (no false negatives) of the employed control dependence: the analysis produces no false alarms when the scope function returns for each statement at least all the statements that control-depend on it. An extreme solution is to include all the statements in the program in each scope, in which case sliced causality becomes precisely the classic happen-before relation. As already pointed out in Section 1 and empirically shown in Section 5, such a choice significantly reduces the coverage of analysis. A better solution, still over-conservative, is to use weak dependence when calculating the control 
scopes. If termination information of loops is available, termination-sensitive control dependence can be utilized to provide correct and more precise results. One can also try to use the classic control dependence instead, but one should be aware that false bugs may be reported (e.g., when synchronization is implemented based on "infinite" loops).

\subsection{Data Dependence on Events}

If a change of state(e) may affect state $\left(e^{\prime}\right)$ then we say $e^{\prime}$ has a data dependence on $e$ and write $e \sqsubset_{\text {data }} e^{\prime}$. Formally,

Definition 8. For events e and $e^{\prime}, e \sqsubset_{\text {data }} e^{\prime}$ iff $e<_{\tau} e^{\prime}$ and one of the following holds:

1. $e<e^{\prime}$, type $(e)=$ read and stmt $\left(e^{\prime}\right)$ uses target $(e)$ to compute state $\left(e^{\prime}\right)$;

2. $\operatorname{type}(e)=$ write, $\operatorname{type}\left(e^{\prime}\right)=\operatorname{read}, \operatorname{target}(e)=\operatorname{target}\left(e^{\prime}\right)$, and there is no other $e^{\prime \prime}$ with $e<_{\tau} e^{\prime \prime}<_{\tau} e^{\prime}$, type $\left(e^{\prime \prime}\right)=$ write, and target $\left(e^{\prime \prime}\right)=\operatorname{target}\left(e^{\prime}\right)$;

3. $e<e^{\prime}$, type $\left(e^{\prime}\right)=\operatorname{read}, \operatorname{stmt}\left(e^{\prime}\right) \notin \operatorname{scope}(\operatorname{stmt}(e))$, and there exists a statement $S$ in scope $(\operatorname{stmt}(e))$ such that $S$ can change the value of target $\left(e^{\prime}\right)$.

The first case in this definition encodes the common data dependence. For example, for an assignment $x:=E$, the write of $x$ has data dependence on the reads generated by the evaluation of $E$. The second case in Definition 8 captures the interference dependence [13] in multithreaded programs, saying that a read depends on the most recent write of the same memory location. For instance, in Figure 4 if the observed execution is $C_{1} S_{1} S_{3}$ then the read of $x$ at $S_{3}$ is data dependent on the most recent write of $x$ at $S_{1}$. We treat lock release as a write on the lock and lock acquire as a read. The third case in Definition 8 is more intricate and relates to the relevant dependence in [10]. Assuming another execution of Figure 4, say $C_{1} S_{2} S_{3}$, no data dependence defined in cases 1 and 2 can be found in this run. However, the change of the value of the read of $i$ at $C_{1}$ can potentially change the value of the read of $x$ at $S_{3}$ : if the value of $i$ changes then $C_{1}$ may choose to execute the branch of $S_{1}$, resulting in a new write of $x$ that may change the value of the read of $x$ at $S_{3}$. Therefore, we say that the read of $x$ at $S_{3}$ is data dependent on the read of $i$ at $C_{1}$, as defined in case 3 . Note that although this dependence is caused by a control statement, it can not be caught by the control dependence; for example, the read of $x$ at $S_{3}$ is not control dependent on the read of $i$ at $C_{1}$ since $S_{3} \notin \operatorname{scope}\left(C_{1}\right)$. Aliasing information is needed to correctly compute dependence defined in case 3 , which one can obtain using any available techniques.

An important observation of Definition 8 is that there are no write-write, read-read, read-write data dependencies. Specifically, case 2 only considers the write-read data dependence, enforcing the read to depend upon only the latest write of the same variable. In other words, a write and the following reads of the same variable form an atomic block of events. This captures in a more general setting the work in [20].

\subsection{Slicing Causality Using Relevance}

When checking a trace $\tau$ against a property $\varphi$, not all the events in $\tau$ are relevant to $\varphi$; for example, to check dataraces on a variable $x$, accesses to other variables or function calls are irrelevant. Moreover, the state attributes of some relevant events may not be 
relevant; for example, the particular values written to or read from $x$ for datarace (on $x$ ) detection. We next assume a generic filtering function that can be instantiated, usually automatically, to concrete filters depending upon the property $\varphi$ under consideration:

Definition 9. Let $\alpha$ : Events- $\oplus$ Events be a partial function, called a filtering function. The image of $\alpha$, that is $\alpha$ (Events), is written more compactly Events ${ }_{\alpha}$; its elements are called abstract relevant events, or simply just relevant events. All thread start and exit events are relevant: $\alpha(e)$ defined whenever type $(e)=$ start or type $(e)=$ exit.

Let us assume an arbitrary but fixed property $\varphi$ in what follows. Intuitively, $\alpha(e)$ is defined if and only if $e$ is relevant to $\varphi$; if $\alpha(e)$ is defined, then $\operatorname{key}(\alpha(e))=\operatorname{key}(e)$ for any attribute $k e y \neq$ state, while state $(\alpha(e))$ is either undefined or equal to state $(e)$.

Definition 10. Let $\alpha(\tau)$, written more compactly as $\tau_{\alpha}$, be the trace of relevant events achieved by applying $\alpha$ on events in $\tau$. Let $\xi_{\alpha}$ denote $\xi_{\tau_{\alpha}}$ for simplicity.

This relevance-based abstraction plays a crucial role in increasing the predictive power of our analysis approach: in contrast to the concrete event set $\xi$, the corresponding abstract event set $\xi_{\alpha}$ allows more permutations of abstract events; instead of calculating permutations of $\xi$ and then abstracting them into permutations of $\xi_{\alpha}$ like in traditional happen-before based approaches, we will calculate valid permutations of a slice of $\xi \cup \xi_{\alpha}$ that contains only events (directly or indirectly) relevant to $\varphi$. This slice is defined using the dependence on concrete and abstract events.

Definition 11. All dependence relations are extended to abstract relevant events: If $e</ \sqsubset_{\text {ctrl }} / \sqsubset_{\text {data }} e^{\prime}$ then also $\alpha(e)</ \sqsubset_{\text {ctrl }} / \sqsubset_{\text {data }} e^{\prime}, e</ \sqsubset_{\text {ctrl }} / \sqsubset_{\text {data }} \alpha\left(e^{\prime}\right)$, and $\alpha(e)</ \sqsubset_{c t r l} / \sqsubset_{\text {data }} \alpha\left(e^{\prime}\right)$, whenever $\alpha(e)$ and/or $\alpha\left(e^{\prime}\right)$ is defined;

$\sqsubset_{\text {data }}$ is extended only when state $\left(\alpha\left(e^{\prime}\right)\right)$ is defined.

We next define a novel dependence relation, called relevance dependence, which is concerned with potential occurrences of relevant events. Consider Figure 4 again. Suppose that relevant events include writes of $y$ and $z$. For the execution $C_{1} S_{1} S_{3}$, only one relevant event is observed, namely the write of $z$ at $S_{3}\left(e^{\prime}\right)$, which is not control dependent on the read of $i$ generated at $C_{1}(e)$. Consider now another execution $C_{1} S_{2} S_{3}$; in addition to $e^{\prime}$, a new relevant event will be generated, namely the write of $y$ at $S_{2}$, caused by the different choice made at $C_{1}$. Hence, a change of state $(e)$ may affect the number of generated relevant events. Formally, we define relevance dependence as follows:

Definition 12. For $e \in \xi, e^{\prime} \in \xi_{\alpha}$, we write $e \sqsubset_{\text {rlvn }} e^{\prime}$ iff $e<e^{\prime}, \operatorname{stmt}\left(e^{\prime}\right) \notin \operatorname{scope}(\operatorname{stmt}(e))$, and there is a statement $S \in \operatorname{scope}(\operatorname{stm} t(e))$ that may generate a relevant event.

Intuitively, if $e \sqsubset_{r l v n} e^{\prime}$ then $e^{\prime}$ is not control dependent on $e$, but when state $(e)$ changes, some new relevant events may occur before $e^{\prime}$. This may invalidate some permutations of $\xi_{\alpha}$ since valid permutations should preserve the exact number of relevant events.

Definition 13. Let $\sqsubset$ be the relation $\left(\sqsubset_{\text {data }} \cup \sqsubset_{c t r l} \cup \sqsubset_{\text {rlvn }}\right)^{+}$. If e and $e^{\prime}$ are concrete or relevant events such that $e \sqsubset e^{\prime}$, then we say that $e^{\prime}$ depends upon $e$.

Definition 14. Let $\overline{\xi_{\alpha}} \subseteq \xi \cup \xi_{\alpha}$ be the relevant slice of events, extending $\xi_{\alpha}$ with events $e \in \xi$ such that $e \sqsubset e^{\prime}$ for some $e^{\prime} \in \xi_{\alpha}$. Let $\overline{\tau_{\alpha}}$ be the abstract trace of $\tau$, i.e., the permutation of $\overline{\xi_{\alpha}}$ consistent with $<_{\tau}$. 
Intuitively, $\overline{\xi_{\alpha}}$ contains all the events that are directly or indirectly relevant to the property $\alpha$. Our goal here is to define an appropriate notion of causal partial order on $\overline{\xi_{\alpha}}$ and then to show that any permutation consistent with it is sound. Recall that we fixed a trace $\tau$; in what follows, $\tau^{\prime}$ is used to refer to any arbitrary trace.

Definition 15. Let $<^{\tau} \subseteq \overline{\xi_{\alpha}} \times \overline{\xi_{\alpha}}$ be the relation $(<\cup \sqsubset)^{+}$, which we call the sliced causality (or sliced causal partial order) of $\tau$.

From here on, by "causal partial order" we mean the sliced one. We next show that sliced causality is an instance of the parametric framework in Section 3 .

Definition 16. Let $C_{\alpha}$ : Traces- $\rightarrow \mathcal{P O}$ (Events) be the partial function defined as $C_{\alpha}\left(\tau^{\prime}\right)$ $=\left(\xi_{\tau^{\prime}}, \prec^{\tau^{\prime}}\right)$ for each $\tau^{\prime} \in$ Traces. Let $\mathcal{F}_{\alpha} \subseteq$ Traces be the set of all possible abstract traces: for each $\tau_{\mathcal{F}} \in \mathcal{F}_{\alpha}$, there is some execution generating $\tau^{\prime}$ such that $\overline{\tau_{\alpha}^{\prime}}=\tau_{\mathcal{F}}$.

Theorem 2. $C_{\alpha}$ restricted to $\mathcal{F}_{\alpha}$ is total and a causality operator. That is, for any abstract trace $\tau_{\mathcal{F}} \in \mathcal{F}_{\alpha}$, each liniearization of $<^{\tau_{\mathcal{F}}}$ corresponds to some possible execution of the multi-threaded system.

Proof (Sketch). The first condition of the causality operator definition can be easily verified. The more intricate part is to show that the trace obtained by permuting two consecutive independent events in an abstract trace is in $\mathcal{F}_{\alpha}$, that is, it corresponds to a possible execution. This is achieved by definning partial executions and feasible prefix traces, i.e., (abstract) traces corresponding to partial executions. The technical details of this proof can be found in the companion technical report.

We can therefore analyze the permutations of relevant events consistent with sliced causality to detect potential violations without re-executing the program.

\section{Evaluation}

We implemented a vector clock algorithm for computing sliced causality as part of JPREDICTOR, a prototype tool for concurrency error detection of JAVA programs. To measure the effectiveness of sliced causality in contrast with more conventional happen-before causalities, we also implemented the algorithm in [19] for extracting happen-before causality from JAVA programs. Interested readers are referred to [3] for more details about the algorithm and the implementation of JPREDICTOR. JPREDICTOR has been evaluated on several concurrent programs. Two measurements were used during the evaluation to compare the sliced causality with the conventional happen-before causality, namely the size of the partial order and the prediction capability to detect data races. The results of our evaluation are shown in Table 1

The first part of Table 1 shows the benchmarks that we used, along with their size (LOC abbreviates "lines of code"), number of shared variables (S.V.), and number of threads created during their executions. Banking and Http-Server are two simple examples showing relatively classical concurrent bug patterns discussed in detail in [7]. Elevator, sor, and tsp come from [22]. Daisy is a small highly concurrent file system proposed to challenge and evaluate software verification tools. Raytracer is a program from the Java Grande benchmark suite, and SystemLogHandler and WebappLoader are 
Table 1. Evaluation of sliced causality

\begin{tabular}{|c||c|c|c||c|c||c|c|}
\hline \multicolumn{4}{|c||}{ Benchmarks } & \multicolumn{2}{c||}{ Causality Size } & \multicolumn{2}{c|}{ Races } \\
\hline Program & LOC & S. V. & Threads & H.B. & S.C. & S.C. & H.B. \\
\hline \hline Banking & 150 & 10 & 11 & 18 & 2 & 1 & 1 \\
\hline Http-Server & 170 & 2 & 7 & 22 & 2 & 2 & 1 \\
\hline Elevator & 530 & 20 & 4 & 240 & 2 & 0 & 0 \\
\hline sor & 600 & 42 & 4 & 21 & 8 & 0 & 0 \\
\hline tsp & $1.1 \mathrm{k}$ & 15 & 3 & 5 & 2 & 1 & 0 \\
\hline Daisy & $1.5 \mathrm{~K}$ & 312 & 3 & 41 & 23 & 1 & 0 \\
\hline Raytracer & $1.8 \mathrm{k}$ & 4 & 4 & 7 & 3 & 1 & 1 \\
\hline SystemLogHandler & 320 & 3 & 3 & 2 & 1 & 1 & 0 \\
\hline WebappLoader & $3 \mathrm{k}$ & 10 & 3 & 9 & 5 & 3 & 0 \\
\hline
\end{tabular}

two components of Tomcat 5.0.28. The property under verification is the datarace of the shared variable. The test cases used in experiments were manually generated using fixed inputs. More bugs could be found if more effective test generation techniques were employed, but that was not our objective here.

The second part of the table shows the coverage improvement of the analysis when using sliced causality versus the happen-before causality. A more precise measure of coverage would be the number of all the sound linearizations of the causal partial order; unfortunately, counting sound linearizations is a \#P-complete problem [1], so it may be no easier than the problem of generating them. Note that a fully constrained partial order, that is, a total order, admits only one linearization, while a fully unconstrained partial order, that is, a set, admits an exponential number of linearizations. Extrapolating, even though it should not be taken as an absolute measure in all situations, we can say that the fewer causal dependencies a partial order has, the larger the number of sound permutations; moreover, we can also say that the number of linearizations is exponential in the number of unordered elements in the partial order. This simplistic and admittedly informal reasoning leads us to an important insight: any reduction in the number of causal dependencies may have a significant impact on coverage; in particular, a linear reduction of the number of causal dependencies can lead to an exponential increase in the coverage of the analysis. Since the total orders on the events of each thread are enforced by both sliced causality and happen-before, we only measure the causal dependencies due to direct inter-thread communication. Therefore, the following dependencies are counted, their number declared the size of the causality, and then used as a measurement metric: $e_{1} \sqsubset e_{2}, \operatorname{thread}\left(e_{1}\right) \neq \operatorname{thread}\left(e_{2}\right)$, and there is no $e_{3}$ such that $e_{1} \sqsubset e_{3}$ and $e_{3} \sqsubset e_{2}$. Our experiments illustrate that sliced causality is significantly smaller than the conventional happen-before, indicating a magnificent increase in the number of covered potential executions.

The third part of Table 1 directly compares the effectiveness of race detection using sliced causality versus happen-before. The first column in this part is the number of races detected by sliced causality, while the second column gives the number of races detected by the standard, unsliced happen-before causality using the same execution traces. As expected, sliced causality is more effective in detecting dataraces, since it covers more potential runs. Even though, in theory, the standard happen-before 
technique may also be able to detect, through many executions of the system, the errors detected from one run using sliced causality, we were not able to find any of the races in some programs, e.g., in tsp and Tomcat, without enabling the sliced causality. Moreover, in these experiments, sliced causality did not produce any false alarms and, except for Tomcat, it found all the previously known dataraces. For Tomcat, two bugs were revealed from the detected dataraces, both of which had been submitted to and confirmed by the developers of Tomcat. More details can be found in [3].

\section{Conclusion}

We presented a parametric approach to happen-before causal partial orders, which facilitates defining and proving correctness of happen-before relations. Existing variants of happen-before causalities can be obtained as instances of this parametric framework. A novel causal partial order relation, called sliced causality, was also defined and shown correct within our parametric framework. Sliced causality employs static and dynamic analysis to filter out unnecessary dependencies on events in order to improve the coverage of analysis without losing soundness. Evaluation shows that sliced causality can significantly increase the coverage of concurrent program analysis and testing.

\section{References}

1. Brightwell, G., Winkler, P.: Counting linear extensions is \#p-complete. In: Annual ACM symposium on Theory of computing (STOC) (1991)

2. Chandy, K.M., Lamport, L.: Distributed snapshots: determining global states of distributed systems. ACM Trans. Comput. Syst. 3(1), 63-75 (1985)

3. Chen, F., Roşu, G.: Predicting concurrency errors at runtime using sliced causality. Technical Report UIUCDCS-R-2005-2660, Department of Computer Science at UIUC (2005)

4. Chen, F., Roşu, G.: Parametric and sliced causality. Technical Report UIUCDCS-R-20072807, Department of Computer Science at UIUC (2007)

5. Chen, F., Roşu, G.: Parametric and termination-sensitive control dependence - extended abstract. In: International Static Analysis Symposium (SAS) (2006)

6. Cooper, R., Marzullo, K.: Consistent detection of global predicates. In: ACM/ONR workshop on Parallel and distributed debugging (PADD) (1991)

7. Farchi, E., Nir, Y., Ur, S.: Concurrent bug patterns and how to test them. In: International Parallel and Distributed Processing Symposium (IPDPS) (2003)

8. Ferrante, J., Ottenstein, K.J., Warren, J.D.: The program dependence graph and its use in optimization. ACM Trans. Program. Lang. Syst. 9(3), 319-349 (1987)

9. Flanagan, C., Godefroid, P.: Dynamic partial-order reduction for model checking software. In: ACM SIGPLAN symposium on Principles of programming languages (POPL), ACM Press, New York (2005)

10. Gyimothy, T., Beszedes, A., Forgacs, I.: An efficient relevant slicing method for debugging. In: ACM SIGSOFT Symposium on Foundations of Software Engineering (FSE), ACM Press, New York (1999)

11. Hoogeboom, H.J., Rozenberg, G.: Dependence graphs. In: Diekert, V., Rozenberg, G. (eds) The Book of Traces, pp. 43-67. World Scientific (1995)

12. Horwitz, S., Reps, T.W.: The use of program dependence graphs in software engineering. In: International Conference on Software Engineering (ICSE) (1992) 
13. Krinke, J.: Static slicing of threaded programs. In: Workshop on Program Analysis for Software Tools and Engineering (PASTE) (1998)

14. Lamport, L.: Time, clocks, and the ordering of events in a distributed system. Comm. of ACM 21(7), 558-565 (1978)

15. Netzer, R.H.B., Miller, B.P.: Improving the accuracy of data race detection. In: ACM SIGPLAN symposium on Principles and practice of parallel programming (PPoPP), ACM Press, New York (1991)

16. O'Callahan, R., Choi, J.-D.: Hybrid dynamic data race detection. In: ACM SIGPLAN Symposium on Principles and Practice of Parallel Programming (PPoPP), ACM Press, New York (2003)

17. Podgurski, A., Clarke, L.A.: A formal model of program dependences and its implications for software testing, debugging, and maintenance. IEEE Transactions on Software Engineering 16(9), 965-979 (1990)

18. Sen, A., Garg, V.K.: Detecting temporal logic predicates in distributed programs using computation slicing. In: Proceedings of the Seventh International Conference on Principles of Distributed Systems (OPODIS) (2003)

19. Sen, K., Roşu, G., Agha, G.: Runtime safety analysis of multithreaded programs. In: ACM SIGSOFT Symposium on Foundations of Software Engineering (FSE), ACM Press, New York (2003)

20. Sen, K., Roşu, G., Agha, G.: Detecting errors in multithreaded programs by generalized predictive analysis of executions. In: Steffen, M., Zavattaro, G. (eds.) FMOODS 2005. LNCS, vol. 3535, Springer, Heidelberg (2005)

21. Stoller, S.D., Unnikrishnan, L., Liu, Y.A.: Efficient detection of global properties in distributed systems using partial-order methods. In: Emerson, E.A., Sistla, A.P. (eds.) CAV 2000. LNCS, vol. 1855, Springer, Heidelberg (2000)

22. von Praun, C., Gross, T.R.: Object race detection. In: ACM SIGPLAN Conference on Object Oriented Programming, Systems, Languages, and Applications (OOPSLA), ACM Press, New York (2001) 